JOURNAL OF INTEGRAL EQUATIONS

AND APPLICATIONS

Volume 6, Number 1, Winter 1994

\title{
TWO REMARKS ON SPECTRAL APPROXIMATIONS FOR WIENER-HOPF OPERATORS
}

\author{
A. BÖTTCHER AND H. WIDOM
}

\begin{abstract}
In this note we give the answers to two questions posed recently by P.M. Anselone and I.H. Sloan in this journal.
\end{abstract}

The Wiener-Hopf integral operator $W(a)$ induced by a complexvalued function $a \in L^{\infty}(\mathbf{R})$ is the bounded operator acting on $L^{2}(0, \infty)$ by the rule

$$
(W(a) \varphi)(t)=\frac{1}{2 \pi} \int_{-\infty}^{\infty} e^{-i x t} a(x) \int_{0}^{\infty} e^{i x s} \varphi(s) d s d x, \quad t>0
$$

If

$$
a(x)=c+\int_{-\infty}^{\infty} e^{i x t} k(t) d t, \quad x \in \mathbf{R}
$$

with $c \in \mathbf{C}$ and $k \in L^{1}(\mathbf{R})$, then $W(a)$ can also be written in the form

$$
(W(a) \varphi)(t)=c \varphi(t)+\int_{0}^{\infty} k(t-s) \varphi(s) d s, \quad t>0
$$

The finite sections $W_{\tau}(a), \tau>0$, of $W(a)$ are the compressions $P_{\tau} W(a) \mid \operatorname{Im} P_{\tau}$, where $P_{\tau}: L^{2}(0, \infty) \rightarrow L^{2}(0, \tau)$ is defined by $\left(P_{\tau} \varphi\right)(t)=\varphi(t)$ for $0<t<\tau$ and $\left(P_{\tau} \varphi\right)(t)=0$ for $t>\tau$. Thus, in case $a$ is of the form (1), we have

$$
\left(W_{\tau}(a) \varphi\right)(t)=c \varphi(t)+\int_{0}^{\tau} k(t-s) \varphi(s) d s, \quad 0<t<\tau .
$$

\footnotetext{
Received by the editors on June 20, 1993.

Research of the first author supported by the Alfried Krupp Förderpreis für junge Hochschullehrer of the Krupp Foundation.

Research of the second author supported by National Science Foundation grant DMS-9216103.
}

Copyright (C)1994 Rocky Mountain Mathematics Consortium 
The comparison of the spectral properties of $W(a)$ and $W_{\tau}(a)$ as well as the discrete analogue of this problem, the comparison of the spectra of an infinite Toeplitz matrix and its finite sections, has been the subject of extensive investigations since at least the sixties (see [1-20], to cite only a few selected works).

One of the questions arising in this connection is the determination of the limit set of the spectra of $W_{\tau}(a)$ as $\tau \rightarrow \infty$ : find the set $B(a)$ of all $\lambda \in \mathbf{C}$ such that $\lambda=\lim \lambda_{n}$ with $\lambda_{n} \in \operatorname{sp} W_{\tau_{n}}(a)$ and $\tau_{n} \rightarrow \infty$. Another question is how $B(a)$ is related to the spectrum of $W(a)$. Both questions turn out to be very difficult in general, but there are two "extremal" cases in which an answer is available. These two cases are the situations in which $a$ is real-valued or rational.

If $a$ is real-valued, then the spectrum sp $W(a)$ of $W(a)$ is equal to the closed interval $[m, M]$, where

$$
m=\underset{x \in \mathbf{R}}{\operatorname{essinf}} a(x), \quad M=\underset{x \in \mathbf{R}}{\operatorname{ess} \sup } a(x),
$$

and it is easy to show that $B(a)$ also coincides with this interval (see [18] for the Toeplitz case). Since this seems unexpectedly not to be widely known (e.g., in [2] it was conjectured that this occurs if $a$ is of the form (1) and a proof was given under the additional hypothesis that $a-c$ be in $L^{1}(\mathbf{R})$ ), we present a proof here.

Theorem 1. Let $a \in L^{\infty}(\mathbf{R})$ be real-valued. Then $\operatorname{sp} W_{\tau}(a) \subset$ $\operatorname{sp} W(a)$ for every $\tau>0$, and given any $\varepsilon>0$, there is some $\tau_{0}>0$ such that $\operatorname{sp} W(a)$ is contained in the $\varepsilon$-neighborhood of $\operatorname{sp} W_{\tau}(a)$ for every $\tau>\tau_{0}$. In particular, $B(a)=\operatorname{sp} W(a)$.

Proof. We first show that $\operatorname{sp} W_{\tau}(a) \subset \operatorname{sp} W(a)$ for every $\tau>0$, which implies that $B(a) \subset \operatorname{sp} W(a)$. So let $\lambda \notin \operatorname{sp} W(a)=[m, M]$. Then $a-\lambda$ is a sectorial function, that is, its essential range is contained in some open half-plane whose boundary passes through the origin. It follows that there is a number $\gamma \in \mathbf{C} \backslash\{0\}$ such that $\|\gamma(a-\lambda)-1\|<1$, and, since the norm of a Wiener-Hopf operator is at most the norm of its symbol, we obtain

$$
\left\|\gamma\left(W_{\tau}(a)-\lambda I\right)-I\right\|=\left\|W_{\tau}(\gamma(a-\lambda)-1)\right\|<1
$$


from which we infer that $W_{\tau}(a)-\lambda I$ is invertible for every $\tau>0$.

To show the other half of the assertion, assume the contrary, i.e., assume there are a $\lambda \in \operatorname{sp} W(a)=[m, M]$, an $\varepsilon>0$, and a sequence $\left\{\tau_{n}\right\}$ such that $\tau_{n} \rightarrow \infty$ and

$$
(\lambda-\varepsilon, \lambda+\varepsilon) \cap \operatorname{sp} W_{\tau_{n}}(a)=\varnothing
$$

for all $n$. Then $(-\varepsilon, \varepsilon) \cap \operatorname{sp} W_{\tau_{n}}(a-\lambda)=\varnothing$ for all $n$, and the spectral theorem shows that $W_{\tau_{n}}(a-\lambda)$ is invertible and

$$
\operatorname{sp}\left(W_{\tau_{n}}^{-1}(a-\lambda) \mid \operatorname{Im} P_{\tau_{n}}\right) \subset(-1 / \varepsilon, 1 / \varepsilon)
$$

for all $n$. Since $W_{\tau_{n}}^{-1}(a-\lambda) \mid \operatorname{Im} P_{\tau_{n}}$ is self-adjoint, its spectral radius is equal to the norm, whence

$$
\left\|W_{\tau_{n}}^{-1}(a-\lambda) \mid \operatorname{Im} P_{\tau_{n}}\right\|<1 / \varepsilon
$$

for all $n$ and consequently,

$$
\left\|W_{\tau_{n}}(a-\lambda) P_{\tau_{n}} \varphi\right\| \geq \varepsilon\left\|P_{\tau_{n}} \varphi\right\|
$$

for all $\varphi \in L^{2}(0, \infty)$ and all $n$. Taking into account that $P_{\tau_{n}}$ converges strongly to the identity operator as $\tau \rightarrow \infty$, we obtain from (2) the estimate

$$
\|W(a-\lambda) \varphi\| \geq \varepsilon\|\varphi\| \quad \text { for all } \varphi \in L^{2}(0, \infty) .
$$

Because $W(a-\lambda)$ is self-adjoint, we may conclude from (3) that $W(a-\lambda)$ is invertible, which contradicts our assumption that $\lambda$ be in $\operatorname{sp} W(a)$.

Our second remark concerns the case where $a$ is a (bounded) rational function. Without loss of generality one may suppose that $a$ is a proper rational function, i.e., $a(x)=f(x) / g(x)$ with

$$
f(x)=x^{r}+f_{r-1} x^{r-1}+\cdots+f_{0}, \quad g(x)=x^{p}+g_{p-1} x^{p-1}+\cdots+g_{0},
$$

and $0 \leq r<p$. If $\lambda \neq 0$, then

$$
\begin{aligned}
\lambda \in \operatorname{sp} W_{\tau}(a) & \Longleftrightarrow W_{\tau}(a)-\lambda I \text { is not invertible } \\
& \Longleftrightarrow I-(1 / \lambda) W_{\tau}(a) \text { is not invertible } \\
& \Longleftrightarrow \operatorname{det}_{2}\left(I-(1 / \lambda) W_{\tau}(a)\right)=\operatorname{det}_{2} W_{\tau}(1-(1 / \lambda) a)=0,
\end{aligned}
$$


where $\operatorname{det}_{2}$ refers to the (second) regularized determinant of operators of the form identity minus Hilbert-Schmidt operator; note that in the case at hand $(1 / \lambda) a \in L^{2}(\mathbf{R})$, which guarantees that $(1 / \lambda) W_{\tau}(a)$ is Hilbert-Schmidt. We may write

$$
\begin{aligned}
1-(1 / \lambda) a(x) & =(g(x)-(1 / \lambda) f(x)) / g(x) \\
& =\prod_{n=1}^{q+s}\left(x-\xi_{n}(\lambda)\right) /\left(\prod_{l=1}^{q}\left(x+i \rho_{l}\right) \prod_{m=1}^{s}\left(x-i \mu_{m}\right)\right),
\end{aligned}
$$

where $\operatorname{Re} \rho_{l}>0$ and $\operatorname{Re} \mu_{m}>0$, and we index the roots $\xi_{1}(\lambda), \ldots$, $\xi_{q+s}(\lambda)$ by increasing imaginary part, so that

$$
\operatorname{Im} \xi_{1}(\lambda) \leq \operatorname{Im} \xi_{2}(\lambda) \leq \cdots \leq \operatorname{Im} \xi_{q+s}(\lambda) .
$$

Then define

$$
C(a)=\left\{\lambda \in \mathbf{C}: \operatorname{Im} \xi_{s}(\lambda)=\operatorname{Im} \xi_{s+1}(\lambda)\right\} .
$$

Theorem 2. $C(a)$ is a nonempty bounded set, which consists of a finite union of closed analytic arcs. We have $B(a)=\{0\} \cup C(a)$.

Proof. This follows from combining Theorem 5.1 of $[\mathbf{3}]$ with the techniques of Schmidt and Spitzer [17] and Day [5].

Notice that in general $C(a)$ is in no obvious way related to sp $W(a)$.

Example. Let $k(t)=e^{t}$ for $t<0$ and $k(t)=2 e^{-t}$ for $t>0$. Then

$$
a(x)=\int_{-\infty}^{\infty} e^{i x t} k(t) d t=(3+i x) /\left(1+x^{2}\right), \quad x \in \mathbf{R} .
$$

In $[2]$ it was conjectured that $B(a)$ is the union of the circle $\{\lambda \in \mathbf{C}$ : $|\lambda-1 / 12|=1 / 12\}$ and the interval $[3 / 2-\sqrt{2}, 3 / 2+\sqrt{2}]$. In fact, Theorem 2 is all we need to determine $B(a)$.

Indeed, we have

$$
1-\frac{1}{\lambda} a(x)=1-\frac{1}{\lambda} \frac{3+i x}{1+x^{2}}=\frac{\left(x-\xi_{1}(\lambda)\right)\left(x-\xi_{2}(\lambda)\right)}{(x+i)(x-i)}
$$


where

$$
\xi_{1 / 2}(\lambda)=\frac{i}{2 \lambda} \pm \frac{1}{2 \lambda} \sqrt{-4 \lambda^{2}+12 \lambda-1},
$$

and Theorem 2 tells us that a nonzero $\lambda$ belongs to $B(a)$ if and only if

$$
\begin{aligned}
\operatorname{Im} \xi_{1}(\lambda) & =\operatorname{Im} \xi_{2}(\lambda) \Longleftrightarrow \xi_{1}(\lambda)-\xi_{2}(\lambda) \text { is real } \\
& \Longleftrightarrow\left(\xi_{1}(\lambda)-\xi_{2}(\lambda)\right)^{2} \geq 0 \\
& \Longleftrightarrow-4+12(1 / \lambda)-1 / \lambda^{2}=\delta \geq 0 \\
& \Longleftrightarrow \lambda=1 /(6+\sqrt{32-\delta}) \text { or } \lambda=1 /(6-\sqrt{32-\delta}) \quad \text { with } \delta \geq 0 .
\end{aligned}
$$

The parameters $\delta \in[0,32]$ give the two intervals

$$
3 / 2-\sqrt{2} \leq \lambda \leq 1 / 6 \text { and } 1 / 6 \leq \lambda \leq 3 / 2+\sqrt{2},
$$

while $\delta=32+\gamma^{2}, \gamma \geq 0$, gives $\lambda=1 /(6 \pm i \gamma)$, which is readily seen to be a parametrization of the circle $|\lambda-1 / 12|=1 / 12$.

\section{REFERENCES}

1. N.I. Achiezer, The continuous analogue of some theorems on Toeplitz matrices, Amer. Math. Soc. Transl. Ser. 250 (1966), 295-316.

2. P.M. Anselone and I.H. Sloan, Spectral approximations for Wiener-Hopf operators II, J. Integral Equations Appl. 4 (1992), 465-489.

3. A. Böttcher, Wiener-Hopf determinants with rational symbols, Math. Nachr. 144 (1989), 39-64.

4. A. Böttcher and B. Silbermann, Analysis of Toeplitz operators, Springer, Berlin, Heidelberg, New York, 1990.

5. K.M. Day, Toeplitz matrices generated by the Laurent series expansion of an arbitrary rational function, Trans. Amer. Math. Soc. 206 (1975), 224-245.

6. H. Dym and S. Ta'assan, An abstract version of a limit theorem of Szegö, J. Funct. Anal. 43 (1981), 294-312.

7. I. Gohberg and I.A. Feldman, Convolution equations and projection methods for their solution, Amer. Math. Soc. Transl. Math. Monographs 41, Providence, R.I., 1974.

8. I. Gohberg, M.A. Kaashoek and F. van Schagen, Szegö-Kac-Achiezer formulas in terms of realization of the symbol, J. Funct. Anal. 74 (1987), 24-51.

9. U. Grenander and G. Szegö, Toeplitz forms and their applications, Univ. Calif. Press, Berkeley and Los Angeles, 1958.

10. I.I. Hirschman, Jr., On a formula of Kac and Achiezer, J. Math. Mech. 16 (1966), 167-196.

11. - The spectra of certain Toeplitz matrices, Ill. J. Math. 11 (1967), 145-159. 
12. M. Kac, Toeplitz matrices, translation kernels, and a related problem in probability theory, Duke Math. J. 21 (1954), 501-509.

13. A.V. Kozak, On the reduction method for multidimensional discrete convolutions, Mat. Issled. 8 (29) (1973), 157-160 [Russian].

14. Yu.I. Linnik, The multidimensional analogue of the limit theorem of Szegö, Math. USSR-Izv. 9 (1975), 1323-1332.

15. L. Reichel and L. Trefethen, Eigenvalues and pseudo-eigenvalues of Toeplitz matrices, Linear Algebra Appl. 162-164 (1992), 153-185.

16. S. Prössdorf, Some classes of singular equations, North-Holland, 1978.

17. P. Schmidt and F. Spitzer, The Toeplitz matrices of an arbitrary Laurent polynomial, Math. Scand. 8 (1960), 15 -38.

18. H. Widom, Toeplitz matrices, Studies in Real and Complex Analysis (I.I. Hirschman, Jr., ed.), M.A.A. Stud. Math. 3 (1965), 179-209.

19. - Asymptotic expansions for pseudodifferential operators on bounded domains, Springer, New York, 1985.

20. - Eigenvalue distribution of nonselfadjoint Toeplitz matrices and the asymptotics of Toeplitz determinants in the case of nonvanishing index, Oper. Theory Adv. Appl. 48 (1990), 387-421.

Fachbereich Mathematik, TU Chemnitz-Zwickau, PSF 964, 09009 ChemNITZ, Germany

Department of Mathematics, University of California, Santa Cruz, CA 95064 